\title{
Human-Centric Functional Modeling and Condensed Matter Physics
}

\author{
Andy E. Williams ${ }^{1}$, Emir Haliki², \\ ${ }^{1}$ Nobeah Foundation, Nairobi, Kenya, awilliams@,nobeahfoundation.org \\ ${ }^{2}$ Hege University, Department of Physics, emir.haliki@ege.edu.tr
}

\begin{abstract}
The newly emerging sciences of Human-Centric Functional Modeling provides an approach towards modeling systems that is hypothesized to maximize human capacity to understand and navigate complexity in those systems. This paper provide an overview exploring how Human-Centric Functional Modeling might be applied in condensed matter physics, and how this increase in capacity to understand complexity might be achieved.
\end{abstract}

\section{Introduction}

It stands to reason that if there are different models for physical systems, there might be some models that maximize our collective capacity to understand those systems. Assume that in a population P, some subset of individuals $\mathrm{N}_{\mathrm{i}}$ understands a given model " $\mathrm{i}$ ", and that this learning can be acquired by a person " $\mathrm{j}$ " in some time $\mathrm{T}_{\mathrm{ij}}$. Assume also that a person " $\mathrm{j}$ " with an understanding of any model " $\mathrm{j}$ " takes some time $\mathrm{T}_{\mathrm{ijk}}$ to learn model " $\mathrm{k}$ ", and that both the time required for that person to learn their first model "i", to learn their second model " $j$ ", and the time available $A_{j}$ for that person to learn any model all have some mean value and some smooth (continuous) distribution about that mean value. Then by the Extreme Value Theorem [1] the total number of people with a correct understanding of the system has a maximum. Through simple deduction we can see that under these assumptions this maximum occurs where the model " $i$ " is correct, where the time required to learn that model is at its minimum, and where everyone learns that single simplest model.

However, defining a single universal model of any system requires special conditions be present. It seems reasonable that to maximize our ability to spread an understanding of a system we must minimize the number of conceptual frameworks that we must understand and apply in order to understand that system. This suggests that if we could all see the world in terms of the same conceptual framework, we could maximize our ability to spread understanding of a given system everywhere [2]. The only framework that every human being has in common is the human organism itself. HumanCentric Functional Modeling attempts to represent this human system as well as all of this existence that can be perceived by this human system in terms of mathematical spaces that are innate to the way every single human being on earth perceives.

\section{Human-Centric Functional Modeling}

In Human-Centric Functional Modeling living systems such as the human organism as well as systems comprised of inanimate matter are modelled as having a set of observable behaviours (functions) through which they transition from one functional state to another. These states form a "functional state space" which the system acting in that domain moves through. All the states accessible through those functions (i.e. all the states in the "functional state space") then reflect all possible behaviors of the system, which is confined to the states accessible within a given domain of behaviour (i.e. all states belong to some common category).

These functional state spaces are represented in terms of a graph containing a network of nodes representing the functional states, connected by edges representing the interactions between those states. It has been claimed that "all Complex Systems can be represented and described as networks" 
[3], in which case a graph of nodes that interact through edges describes every region in the physical universe that interacts through forces. If so, from the perspective of Human-Centric Functional Modeling, matter in any subset of the universe, whether the matter in a teacup, the matter in a chair, or the matter in an entire galaxy, can be represented as moving through its own functional state space in a way that solves the general problem of optimizing the fitness of its state in representing the external forces on it. This is general problem-solving ability in that functional state space.

A valid representation of the conceptual space as a functional state space representing all concepts and reasoning must provide a completely self-contained representation of the human meaning of those concepts and reasoning that is capable of storing all the properties of each concept or reasoning process at any scale without the need for any external reference or translation table, so that any cognitive process at any scale can be executed by the same cognitive system. If all matter can also be represented in terms of functional state spaces, this implies that any such representation is also a "semantic representation" in that in order to represent all interactions with the external environment this model must provide a completely self-contained representation of the meaning of that environment. This requires that each node in the functional state space define all the properties that each interaction force acts on, so that calculating the evolution of that matter as a result of all interactions is possible.

This approach is human-centric because it enables cognition to be understood in terms of the cognitive system's motion through its functional state space (the space of concepts or the "conceptual space"). This motion can in turn be understood through first person observation (observations that can be validated within the individual's observation of their own awareness), as opposed to observations requiring third party validation (i.e. measurements made by external tools or assessing validity in terms of consistency with some theory defined by some third party). The fact that first person observations can potentially be related to this functional state space as a mathematical space also means that these first person observations might be used to define the properties of systems like cognition mathematically, including properties like general problem-solving ability or like the complexity of a problem for the cognitive system. In understanding human systems like cognition in which many of the functions cannot be externally measured, a first person approach is essential. For example, through introspection we can validate that we are able to create or forget concepts, therefore our own conceptual space is dynamic (concepts can be added or removed), which might be true for the functional states in all functional state spaces.

As mentioned, from the perspective of Human-Centric Functional Modeling a problem with regards to understanding any system is the lack of at path through functional state space from one functional state to another. A solution is the process which provides that path. In other words, from the human-centric perspective, the universe can potentially be represented as being comprised of a distributed cognition that computes the evolution of matter in each region of space. The awareness of these interactions propagates in a well-defined way, so the universe also can be represented as being comprised of a distributed awareness (consciousness), but we will ignore this distributed consciousness for now as understanding this distributed cognition is sufficient to communicate the concept of complexity from a human-centric perspective as contemplated in this paper.

Through this first person approach, definitions of the properties of systems in general can potentially be deduced through first person observation of our own cognitive system. In the conceptual space hypothesized to be occupied by our cognitive system, any problem can potentially have many solutions, each of which traces its own path through the conceptual space. 


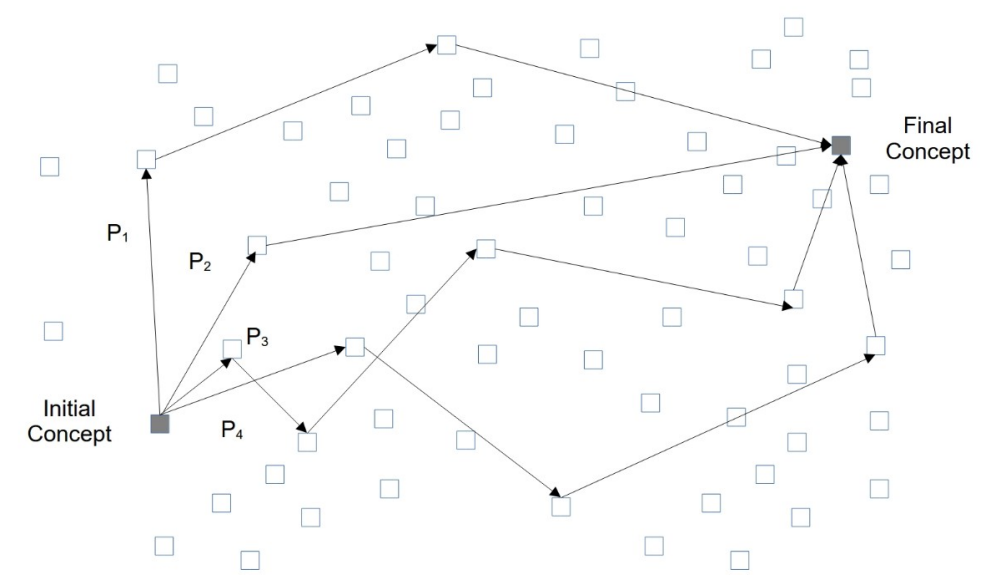

Figure 1: Multiple reasoning paths to the same conclusion might exist in conceptual space.

Though multiple reasoning paths exist, each of those reasoning paths does not necessarily represent truth. Any given path might be complete and consistent in conceptual space, but at the same time not be "true". Consider the truth of reasoning to be a signal. Concepts are output transmitted by reasoning processes of the cognition. Therefore, representing reasoning processes as channels, then according to information theory the ability of any truth signal to be transmitted depends on the characteristics of these processes as channels (i.e. their ability to transmit signal without error). In conceptual space a generalization of a concept is a second concept that encloses a larger region in conceptual space. Any reasoning process can be generalized by generalizing the concepts involved. As the concepts in the reasoning are generalized, the generalized reasoning connecting them becomes a broader path through conceptual space that includes a greater and greater number of more specific reasoning paths.

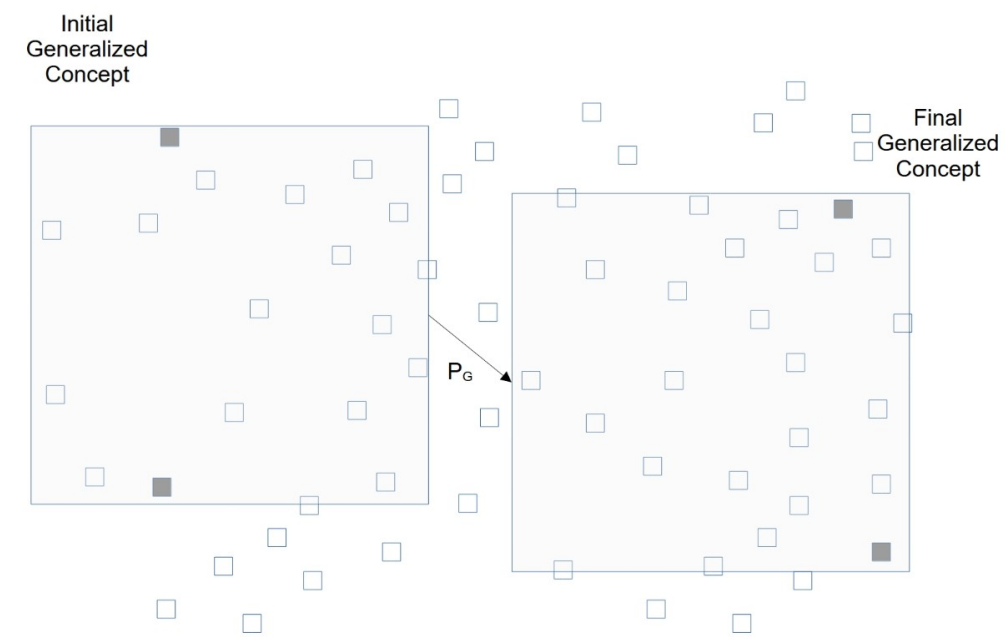

Figure 2: Generalized reasoning process.

From a functional perspective the truth of reasoning is what it functions to achieve. Assume that each of those sets of reasoning paths function to increase the fitness of the cognitive system if they are complete and self-consistent, and that they function to decrease the fitness of the cognitive system if they are incomplete or inconsistent. Then a high degree of completeness and consistency through all of the different reasoning paths that a given reasoning can be generalized to represents a signal of "truth", and the opposite represents a signal of "falsehood". 
The position of concepts in conceptual space is defined by the relationships that define those concepts. Therefore the adoption of one reasoning process by the cognitive system as opposed to another will move the ending concept in conceptual space. Truthful reasoning will then result in a different motion of the ending concept as compared to untruthful reasoning. There are then at least two ways to detect truth in conceptual space: the truth of any reasoning process (the degree to which it transmits truth) can be determined by whether it leads to reasoning that is complete and self-consistent when generalized to other contexts; and the truth of any reasoning process can be determined by the position in conceptual space of the concept that is output by that reasoning.

Once truth has been defined as a signal transmitted with differing degrees of falsehood by each reasoning process (the first method), and once that signal can be detected as a difference in position in conceptual space (the second method), then truth becomes a property that can potentially be detected at exponentially greater speed and scale. In the second case this entails using AI to detect familiar patterns of truth (or untruth) using type 1 (intuitive) reasoning, or using procedural programs to detect logic (type 2 or rational methodical reasoning) that represents truth, and by using General Collective Intelligence or GCI [4] to select which is truth detection mechanism (type 1 or type 2 thinking) is optimal in each context. In either case this introduces the possibility of exponentially greater general problem-solving ability, and exponentially greater ability to solve any problem in general, which might be reflected in an exponentially greater ability to achieve impact in any research discipline [5].

Using these definitions of truth, the truth of any property of cognition might potentially be evaluated. The complexity of a solution is hypothesized to be the distance through functional state space that is navigated by the solution, multiplied by the linear density of functional states the solution moves through. The complexity of a problem is assumed be be the complexity of the least complex solution. General problem-solving ability is hypothesized to be the volume of that functional state space the system can navigate to a solution per unit time, multiplied by the density of functional states the system must move through. The complexity of a system is hypothesized to be the volume of that functional state space the system can navigate, multiplied by the density of functional states the system must move through.

\section{Modeling the Evolution of Physical Systems}

In Human-Centric Functional Modeling it is assumed that all behaviors of a system can be understood in terms of its functional state space. Assume that all the potentially complex behaviors in a given domain can be represented as some combination of a very simple set of behaviors (functions or operations). In other words, assume that any network of functional states can be constructed with some sequence of only a few different types of operations. In mathematical terms a set of operations is said to "span" a space when any point in that space can be represented as some combination of those operations. It has been hypothesized that a set of four operations can "span" the network of functional states representing conceptual space [8]. Representing the mind as moving through its own functional state space (again, a space of concepts or a "conceptual space"), this means that if a set of four operations spans this space, those four operations can potentially represent any reasoning or understanding process connecting concepts, and can therefore define any concept. Since there are four known forces, this might be true of the functional state space representing matter as well.

Any system with a stable set of repeatable functions also must stay within a bounded region of a "fitness space" that describes the fitness of the system to execute its functions. A change in fitness of the system occurs as a result of some action, that is, as a result of following some path, in functional state space. Defining a generalized "fitness space" for all problems, such as defined by the three dimensions of target value of fitness, actual value of fitness, and predicted value of fitness, then the 
path through this fitness space must stay within a bounded region of fitness space. In this sense the motion in fitness space must be stable globally throughout the fitness space, despite potentially being chaotic in functional state space due to random interactions with the environment.

From the perspective of Human-Centric Functional Modeling, randomness due to uncertainty, particularly at quantum scales, might alternatively be viewed as the laws of physics fluctuating within a fitness space that might be larger or smaller depending on the degree of uncertainty. Momentum or any other property might randomly fluctuate according to uncertainty, but must be conserved over time from quantum scales to cosmic scales.

Consider a single particle. According to one of potentially other approaches [9], [10] to quantum mechanics, a particle is represented by a wave function that is a solution to schrodinger's equation [11]:

$$
i \hbar \frac{\partial}{\partial t}|\psi(t)\rangle=\hat{H}|\psi(t)\rangle
$$

This solution will have some uncertainty in position and momentum. At higher energies the relative uncertainty is less and particles act more like waves. At lower energies the relative uncertainty is greater and particles act more like waves.

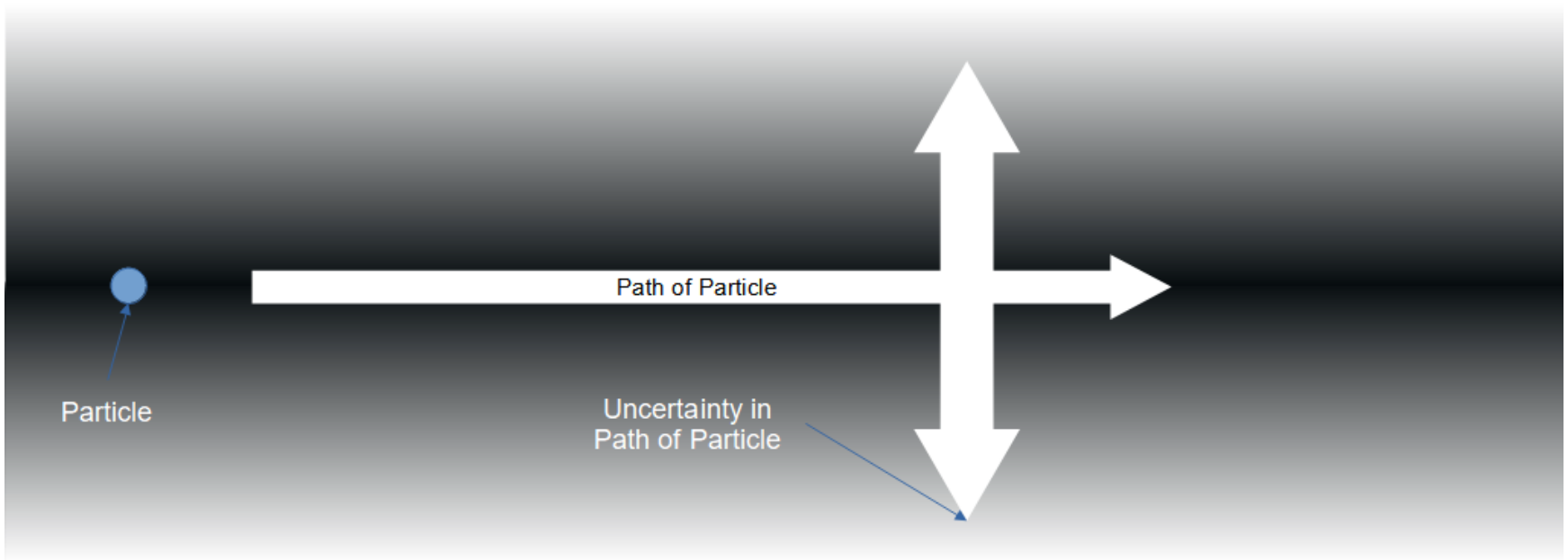

Figure 3: Conceptual representation of the uncertainty in the path of a particle.

When interacting with a field, the wave function must collapse to a specific value for each property with which it interacts. The minimum time interval that is resolvable is a single Planck unit of time. So wave functions don't continually collapse, but instead must collapse at those minimum intervals at most. However, due to uncertainty, the value of any property governed by uncertainty, including the time interval at which the wave function collapses, is not deterministic at each time when that wave function collapse might occur. 


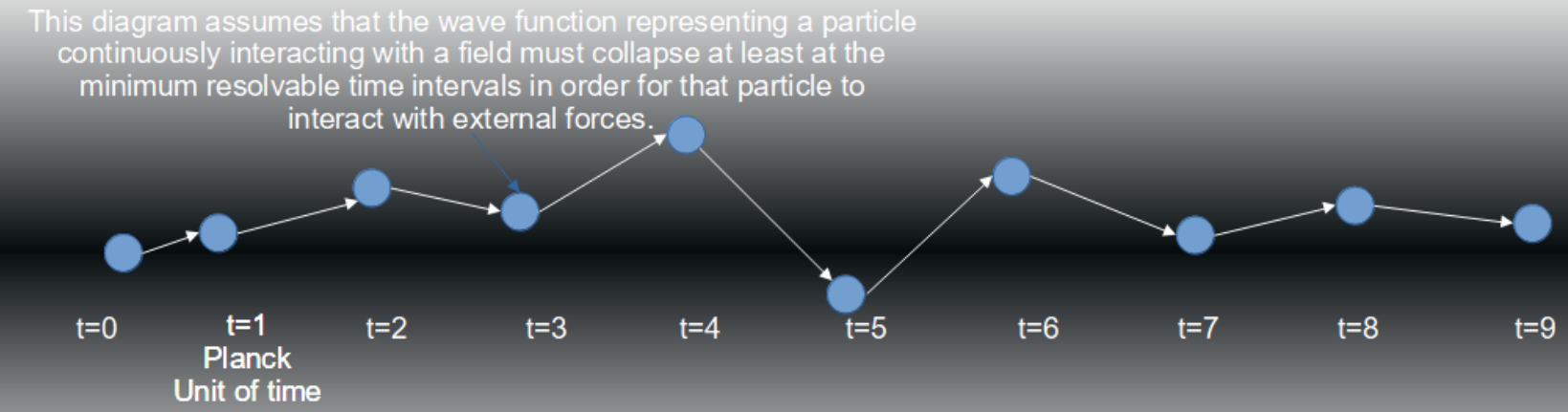

Figure 4: Collapse of the wave function representing a particle continuously interacting with a field. 
As a result, any particle might have a target trajectory determined by the current model of physics if it's momentum and other properties are to be conserved from those at $t=0$. It might also have a projected trajectory from its motion and other properties in the last time slice $t=7$. And it might have an actual trajectory at the current time $\mathrm{t}=8$.

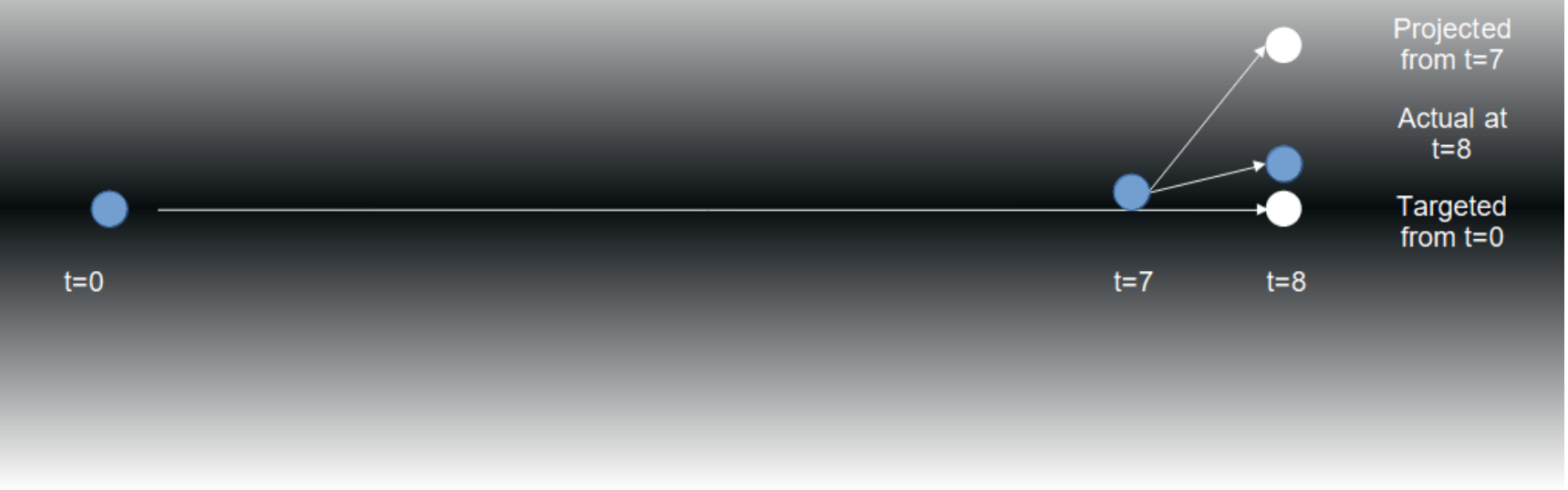

Figure 5: Projected, actual, and targeted positions of the particle from the perspective of HumanCentric Functional Modeling.

In Human-Centric Functional Modeling fitness space is defined by fitness of the projected, actual, and targeted states to represent the particle. The state of the particle must stay in a bounded region in fitness space if momentum and other physical properties are to be conserved.

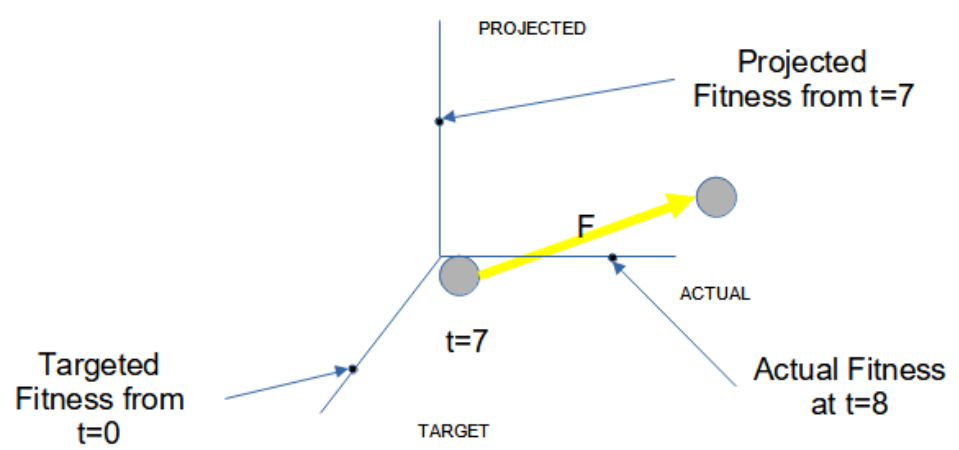

Figure 6: Fitness space of the particle from the perspective of Human-Centric Functional Modeling.

For any theoretical model consisting of set of theories of physics, any region of matter interacts with any other region of matter through the forces defined by that theoretical model. From the perspective of Human-Centric Functional Modeling any set of theories of physics that are complete must keep the state of any given region of matter within a bounded region of fitness space if properties are to be conserved. This implies dynamics that are globally stable throughout fitness space across all the forces with which matter or energy might interact.

One set of equations that potentially satisfies this stability in the three dimensions of fitness space in order to allow properties to be conserved over time despite fluctuations due to uncertainty are the 
Lorenz equations governing convection [14], which for certain values of their parameters form a strange attractor.

$$
\begin{aligned}
& \frac{d x}{d t}=\sigma(y-x) \\
& \frac{d y}{d t}=x(\rho-z)-y \\
& \frac{d z}{d t}=x y-\beta z
\end{aligned}
$$

The relationship between the dynamics in functional state space and the dynamics in fitness space is yet to be explored.

\section{Simulating the Evolution of Physical Systems}

Using Human-Centric Functional Modeling to model matter in terms of a functional state space, the equations of physics can potentially be applied to that functional state space to calculate the evolution of that matter over time. Assume the functional state of the system at the current point in time is $\mathrm{C}_{0}$. The probability of being in a functional state $C_{i}$ at the next point in time is given by some probability tensor $\mathbf{P}$ multiplied by the vector of transition processes $\mathbf{C}$, where $\mathbf{P}$ specifies the probability of any process $P_{i}$ and where each element $C_{i}$ in the vector $\mathbf{C}$ is a transition process accessible from the original state $\mathrm{C}_{0}$.

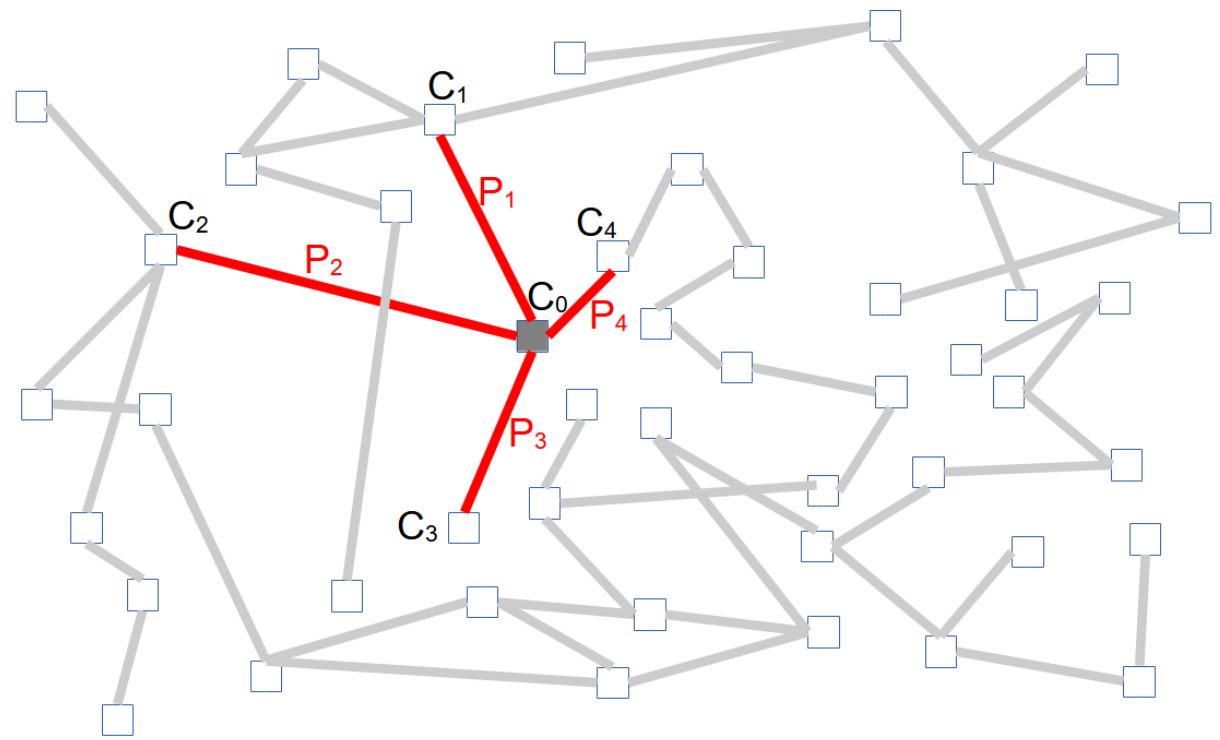

Figure 7: Evolution of a single point $C_{0}$ in functional state space. Any functional state space is represented by a network of nodes representing the functional states, connected by edges representing the processes by which the system transitions from one functional state to another.

The actual evolution of the system might be simulated through selecting transition processes according to these probabilities using a Monte Carlo based algorithm. 


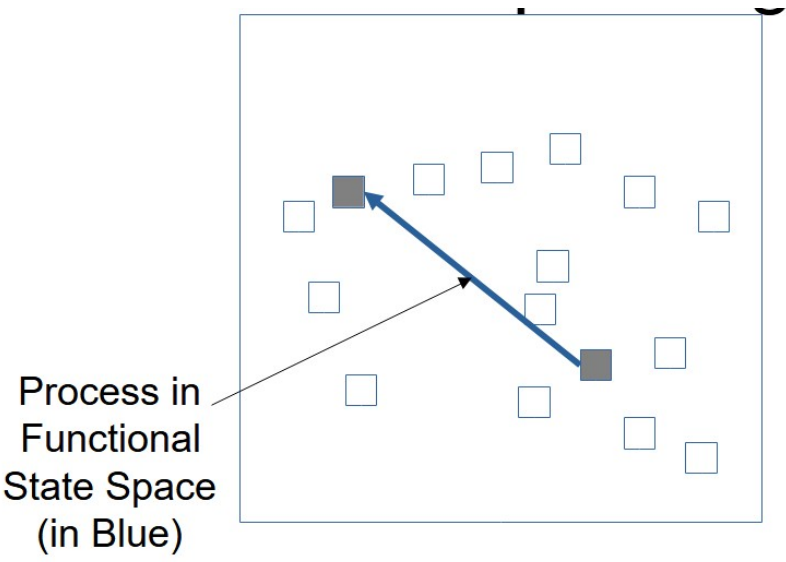

Functional State Space

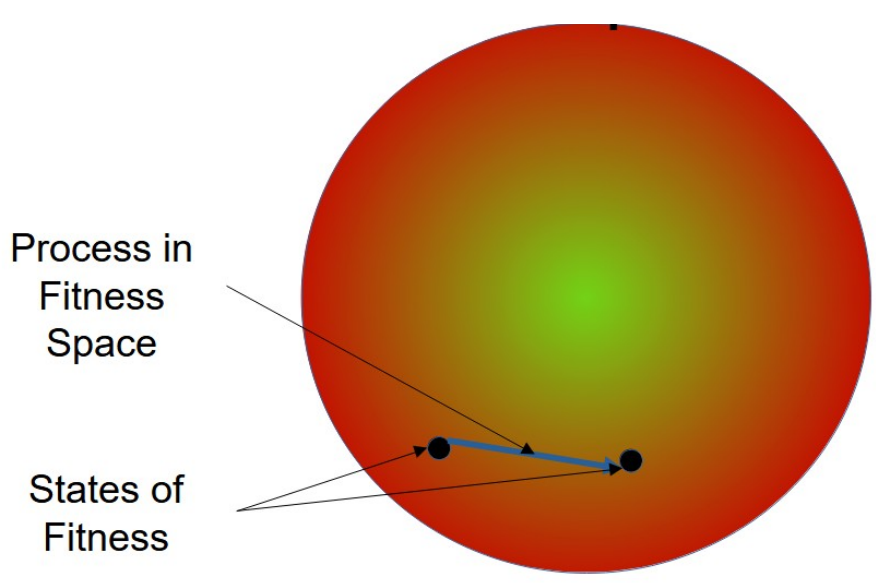

Stable Region of Fitness Space

Figure 8: Path of system in functional state space and corresponding path in fitness space: The evolution of matter can be represented as being a localized system in which transition processes trace a path through the functional state space of the system, while also tracing a corresponding path through the fitness space of the system.

In the case of distributed matter or energy, rather than considering the system as evolving from being in one position in functional state space (represented by the dark square labeled $\mathrm{C}_{0}$ ) to being in a different position in functional state space, every node in the functional state space representing matter or energy in each region is its own system that also evolves. matter are then represented as distributed systems. 


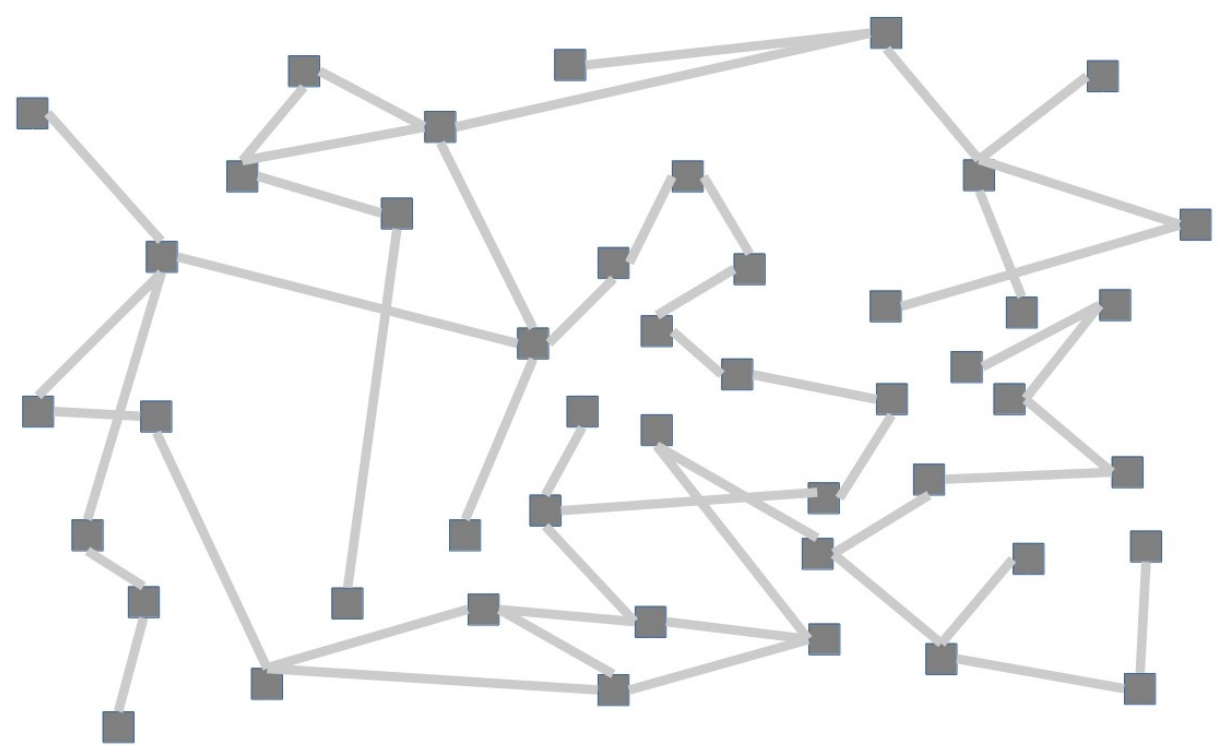

Figure 9: Evolution of all points in functional state space.

In order to simulate the evolution of these distributed systems, each node might be represented as evolving according to its own fitness space. In the classical case, the evolution of each point in functional state space will be deterministic. In the quantum mechanical case the evolution of each point in functional state space will be probabilistic. One area of potential exploration for the future is how the equations of physics can be applied to this functional state space in the classical case. Another area of potential exploration is how the equations of physics can be applied to this functional state space in the quantum mechanical case. In either case this representation in terms of a functional state space might enable the same simulation infrastructure to potentially be reused between simulating cognition and simulating physics. 


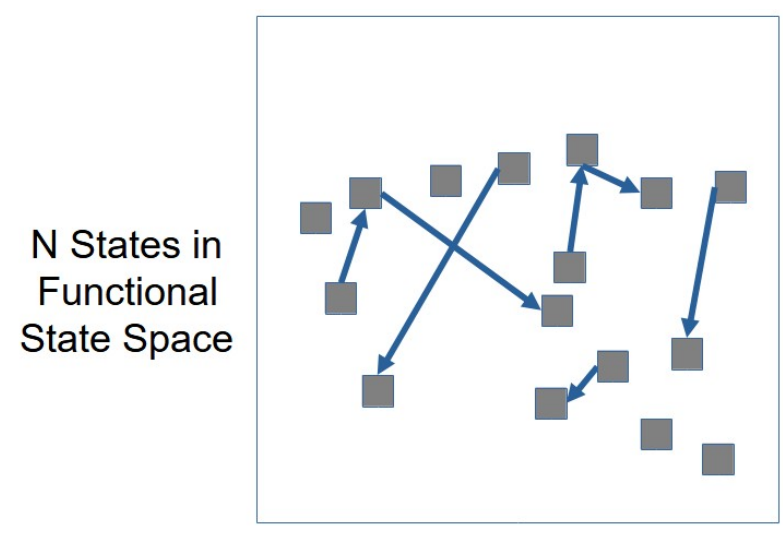

Functional State Space

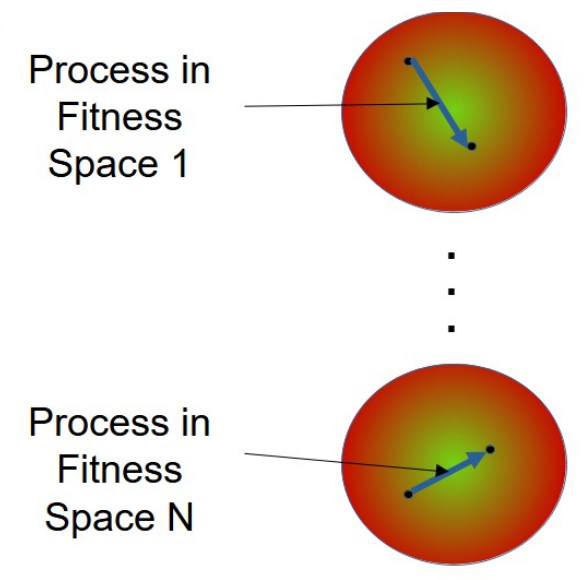

Stable Region of Each Fitness Space "i"

Figure 10: Path of a distributed system in its functional state space and its corresponding path in its fitness spaces: The physical universe can be represented as a distributed system in which each point evolved according to its own fitness space.

From the perspective of Human-Centric Functional Modeling, optimizing outcomes for inanimate matter in some region means optimizing the fitness of its state in representing the external forces on it. Within the domain of any force this optimization requires the ability to solve the problem of responding to any forces in general, or in other words, requires general problem-solving ability within the physical domain. Whether in the real world, or in a virtual representation of the real world that is capable of real world complexity, exercising this ability in the domain of a given force requires the ability to model all dimensions of interaction with the external environment semantically in that this model must be a completely self-contained representation of the meaning of that environment.

From the perspective of Human-Centric Functional Modeling, optimizing outcomes for inanimate matter in all regions also means optimizing the fitness of its state in representing the external forces on it. Within the domain of any force this optimization again requires general problem-solving ability within the physical domain. Whether in the real world, or in a virtual representation of the real world that is capable of real world complexity, exercising this ability in the domain of a given force again requires the ability to model all dimensions of interaction with the external environment semantically. Since every region is part of the external environment of every other region, this requires the ability to model all regions semantically.

\section{Assessing the Validity of the Approach}

Given that the application of Human-Centric Functional Modeling to representing systems in condensed matter physics is only one of a great many applications being explored, the goal of this paper is not to engage in exhaustive study to determine the validity of the approach, but to simply probe the issues to gain some assurance that the questions are valid and also that they have intellectual merit and broader implications, so that those questions might be opened for exploration by other specialists in this discipline. With sufficient collaborations might be formed to enable groups of local researchers to take part in a set of planned Large Scale international Collaborative REsearch (LSCORE) initiatives intended to test and to elaborate the underlying theory and to build components of the General Collective Intelligence infrastructure required to achieve an exponentially greater impact on condensed matter physics research through Human-Centric Functional Modeling. 


\section{Applications}

A human-centric representation (a fully self-contained representation of human meaning) means defining a "semantic model" of physical matter capable of storing all the properties of that matter at any scale without the need for any external reference or translation table, so that any physical effects at any scale can be explored using the same "semantic simulation" infrastructure. While other approaches towards defining semantic representations of physical systems have been proposed [12], HumanCentric Functional Modeling also facilitates the definition of a model of cognition, and as one researcher has stated "it is hard to imagine that one could give a complete theory of semantic representation outside of a complete theory of cognition in general" [13].

With a semantic model any representation of the physical world, every simulation, every computer game, every Computer Aided Design (CAD) tool, every Computer Generated Image (CGI), or every other computer interaction becomes part of a single data format, and every component in every scene can potentially be reused by every other such computer interaction. The ability to decouple every component of every model so that it can potentially be reused in every other simulation, or game, combined with semantic search to identify those opportunities for reuse, and combined with General Collective Intelligence to multiply those opportunities, is expected to exponentially increase opportunities to reuse such components. In this way, every simulation might contribute infrastructure that might be used in all physics research and vice versa, thereby effectively multiplying research funding.

\section{Implications}

Semantically modeling the physical universe so that every experiment can be stored in a single data model, and so that component of every theory in physics can be tested against every component of every other theory for all available data might radically increase our collective capacity to do physics so that all theories can be considered thoroughly, rather than relegating some theories to the fringe without consideration simple due to our lack of capacity to investigate all of them.

Solving the problem of defining a representation for the functional state space of physical systems also solves the problem of defining a representation for the functional state space of the cognitive system (the conceptual space), and solving the problem of defining a representation for the conceptual space has the potential to exponentially increase our collective capacity to address the existential problems (i.e. wicked problems [6]) facing civilization like poverty and climate change. However, some properties of certain functional state spaces, for example distances in conceptual space, are currently unknown. Any properties in conceptual space that are dependent on distances are then also unknown. For example, cognitive complexity, or general problem-solving ability in the cognitive domain (intelligence), are unknown and can only be estimated to the degree that these distances can be.

In the sense that from the perspective of Human-Centric Functional Modeling all systems from cognition to the physical universe fall within the same general model for adaptive problem-solving [?], these systems can potentially be simulated using the same infrastructure but with different rules. The value proposition of participating in a program like LSCORE is then that we are all solving the same abstract problem. Uniting physicists, mathematicians, computer scientists, and others in solving these problems where others in domains such as sustainable development might be less equipped to do so, might potentially multiply impact in all areas pursuing solutions. Some of the tasks remaining in terms of modeling are to: Create a representation of open functional state spaces that can be used to represent the physical universe, conceptual space, or awareness (consciousness) space; define the minimal set of operations that can "span" this functional state space so that a set of functional components 
implementing that set of operations can span all behaviors in that space. Some of the tasks remaining in terms of implementation are to: define a library of implementations for each functional component and define the context in which each implementation is most fit in executing its intended functions.

Finally, some of the tasks remaining in terms of simulation are to: define a simulation for the physical world based on a semantic model (based on a functional state space); define a simulation for the awareness (consciousness) of interactions within the physical world.

\section{Conclusions}

Semantically modeling the physical universe is a massive task. Before this task can be achieved, it's important to first communicate the possibilities that might be presented by Human-Centric Functional Modeling in even this one branch of physics, to encourage others to validate the feasibility of the approach in this and other disciplines, until the value proposition has appears to be present in a large enough range of disciplines to reliably motivate that effort.

\section{References}

[1] Protter, M. H.; Morrey, C. B. (1977). "The Boundedness and Extreme-Value Theorems". A First Course in Real Analysis. New York: Springer. pp. 71-73. ISBN 0-387-90215-5.

[2] Andy E. Williams, A Revolution in Systems Thinking?, Proceedings of the World Organization of Systems and Cybernetics (WOSC) 2021 Congress, Accepted (2021), link

[3] Newman MEJ (2010) Networks: an introduction. Oxford University Press, New York

[4] Williams, A. E., Defining a Continuum from Individual, to Swarm, to Collective Intelligence, to General Collective Intelligence, International Journal of Collaborative Intelligence, in press (2021)

[5] Williams, A. E. (2021, October 2). Using Systems Thinking to Exponentially Increase Impact. In review (2021), Retrieved from osf.io/preprints/africarxiv/dcjyf

[6] Andy E. Williams, Are Wicked Problems a Lack of General Collective Intelligence?, AI \& Society, DOI: $10.1007 / \mathrm{s} 00146-021-01297-8$, In print (2021)

[7] Williams, A. E. (2021, June 13). General Problem-Solving Ability in Natural Systems as a Model for Computation. https://doi.org/10.31730/osf.io/5x3g7

[8] Williams A.E. (2020) A Model for Artificial General Intelligence. In: Goertzel B., Panov A., Potapov A., Yampolskiy R. (eds) Artificial General Intelligence. AGI 2020. Lecture Notes in Computer Science, vol 12177. Springer, Cham. https://doi.org/10.1007/978-3-030-52152-3 38

[9] Herbert S. Green (1965). Matrix mechanics (P. Noordhoff Ltd, Groningen, Netherlands) ASIN : B0006BMIP8.

[10] Weinberg, S. (2002) [1995], Foundations, The Quantum Theory of Fields, 1, Cambridge: Cambridge University Press, ISBN 978-0-521-55001-7

[11] Schrödinger, E. (1926). "An Undulatory Theory of the Mechanics of Atoms and Molecules" (PDF). Physical Review. 28 (6): 1049-1070. 
[12] Lewis, Martha, Dan Marsden, and Mehrnoosh Sadrzadeh. "Semantic Spaces at the Intersection of NLP, Physics, and Cognitive Science." FLAP 7.5 (2020): 677-682.

[13] Griffiths, Thomas L., Mark Steyvers, and Joshua B. Tenenbaum. "Topics in semantic representation." Psychological review 114.2 (2007): 211.

[14] Lorenz, E. N. (1963). Deterministic nonperiodic flow. Journal of atmospheric sciences,20(2):130141. 